\section{Drs. Singh and Magrey reply}

To the Editor:

We thank Paras Karmacharya and colleagues for their letter to the editor ${ }^{1}$, which furthers the discussion about racial differences in patients with ankylosing spondylitis (AS). They highlight that the reported prevalence of AS in African Americans of $8 \%$ in our study is $l_{0}{ }^{2}$. At present the true prevalence of AS in African Americans in the USA is unknown. Based on the US National Health and Nutrition Examination Survey 2009-2010 survey, the overall prevalence estimates of axial spondyloarthritis (axSpA) using the European Spondylarthropathy Study Group criteria is $0.9 \%$ in non-Hispanic blacks between the ages of 20 and 69 years ${ }^{3,4}$, but Karmacharya and colleagues acknowledged that estimates could be unreliable. The number of African Americans was too low to make any definite estimates.

We acknowledge that healthcare databases have substantial variation in estimates of prevalence and incidence of chronic conditions, which limits their interpretability and utility.

The true prevalence of AS in African Americans may have been underestimated in our study because not all the African American patients with AS in the United States may be seeking medical care at the institutes from where Explorys collects the data. Despite these challenges, medical databases offer a unique opportunity that allows rapid measurements and analysis of data to provide critical information pertinent to public health ${ }^{5}$. The Explorys database covers all 50 states, providing a broad regional distribution of source population.

Karmacharya and colleagues presume that the higher values of erythrocyte sedimentation rate and $\mathrm{C}$-reactive protein in the African American patients with AS is due to a confounding bias, and that only African American patients with elevated markers of inflammation may be getting diagnosed with the disease or seeking care at the participating institutes. African American patients with normal markers of inflammation may not be seeking care. We agree that delay in diagnosis is a well-recognized problem in AS, particularly in women and in patients who are HLA-B27negative. However, this similar assumption can also be made for all patients with normal markers of inflammation; otherwise, presuming that only African American patients with higher markers of inflammation seek medical attention signifies an implicit bias.

Based on the findings of our study, we believe that African American patients with AS may have worse inflammation. Another study also revealed higher disease activity in black patients (including African Americans) with $\mathrm{AS}^{6}$. Karmacharya and colleagues presume that delayed diagnosis may be the reason for increased disease activity and inflammation in African Americans, which may be correct, but that is beyond the scope of our study and we have acknowledged the limitation of the Explorys database. Deidentified data at the population level are available and further review of individual records to verify patient history is not possible. Another limitation is the inability to obtain socioeconomic data from Explorys that would have allowed study of whether socioeconomic factors may have contributed to high disease activity. We did not find any significant differences in use of tumor necrosis factor inhibitors between African Americans and whites.

Karmacharya and colleagues also suggested that increased frequency of extraarticular manifestations among African Americans raises the possibility of detection bias because only those African American patients with these typical features and HLA-B27 positivity might have been diagnosed, and that others with subtle symptoms might have been missed. However, the frequency of HLA-B27 positivity was very low $(25 \% ; 2970 / 10,990)$ in the cohort and there was no significant difference between the 2 groups $(26 \%$ whites vs $20 \%$ African Americans, $\mathrm{p}=0.11)^{2}$, so HLA-B27 positivity as a confounder is less likely.

The authors also raised a concern that patients with nonradiographic axSpA (nr-axSpA) may not have been included in the analysis and presumed that African American patients may have a higher prevalence of nr-axSpA. The term $n r$-axSpA originated from 2009 Assessment of Spondyloarthritis international Society (ASAS) classification criteria $^{7}$ and there is no
International Statistical Classification of Diseases and Related Health Problems (ICD)-9 or ICD-10 code for it; hence, US rheumatologists may have used the ICD-9 code 720.0 (AS) for nr-axSpA, so considering that as a detection bias may not be valid ${ }^{8}$.

We completely agree with Karmacharya and colleagues that a population-based epidemiological study with adequate representation of African American patients might provide better insight into the racial prevalence and clinical features of axSpA in this population ${ }^{1}$. Currently, we have no diagnostic criteria for axSpA and rely on classification criteria for large epidemiologic (populations) studies in $\operatorname{axSpA}^{9}$. Classification criteria have high specificity to avoid misclassification but low sensitivity, raising the possibility of underestimation of true prevalence. ASAS classification criteria cannot simply be applied in large epidemiologic (population) studies ${ }^{10}$. There are very few studies that have addressed racial differences in AS and population-based studies are ideal but lack feasibility because of cost and manpower. Our study is the first real-world study, to our knowledge, to look at racial determinants of clinical features, disease activity, and comorbidities in AS in the United States.

MARINA N. MAGREY, MD; DILPREET KAUR SINGH, MD, Case Western Reserve University, MetroHealth Medical Center, Cleveland, Ohio, USA. Address correspondence to Dr. D.K. Singh, Case Western Reserve University, MetroHealth Medical Center, Division of Rheumatology, 2500 MetroHealth Drive, Cleveland, Ohio 44109, USA. E-mail: dilpreetsinghmd@gmail.com

\section{REFERENCES}

1. Karmacharya P, Balls-Berry JE, Davis JM 3rd. True difference or detection bias: racial differences in clinical features and comorbidities in ankylosing spondylitis in the United States. J Rheumatol 2020;47:1153.

2. Singh DK, Magrey MN. Racial differences in clinical features and comorbidities in ankylosing spondylitis in the United States. J Rheumatol 2020;47:835-8

3. Reveille JD, Weisman MH. The epidemiology of back pain, axial spondyloarthritis and HLA-B27 in the United States. Am J Med Sci 2013;345:431-6

4. Reveille JD, Witter JP, Weisman MH. Prevalence of axial spondylarthritis in the United States: estimates from a cross-sectional survey. Arthritis Care Res 2012;64:905-10.

5. Explorys Team. IBM Explorys solutions. Explorys EHR solutions. [Internet. Accessed January 24, 2020.] Available from: www.explorys.com/about-us.html

6. Jamalyaria F, Ward MM, Assassi S, Learch TJ, Lee M, Gensler LS, et al. Ethnicity and disease severity in ankylosing spondylitis a cross-sectional analysis of three ethnic groups. Clin Rheumatol 2017;36:2359-64.

7. Rudwaleit M, van der Heijde D, Landewé R, Listing J, Akkoc N, Brandt J, et al. The development of Assessment of SpondyloArthritis international Society classification criteria for axial spondyloarthritis (part II): validation and final selection. Ann Rheum Dis 2009;68:777-83.

8. Deodhar A, Strand V, Kay J, Braun J. The term "non-radiographic axial spondyloarthritis" is much more important to classify than to diagnose patients with axial spondyloarthritis. Ann Rheum Dis 2016;75:791-4

9. Stolwijk C, Boonen A, van Tubergen A, Reveille JD. Epidemiology of spondyloarthritis. Rheum Dis Clin North Am 2012;38:441-76.

10. Franklin JM, Schneeweiss S. When and how can real world data analyses substitute for randomized controlled trials? Clin Pharmacol Ther 2017;102:924-33.

First Release February 15 2020; J Rheumatol 2020;47:7; doi:10.3899/jrheum.200048 